\title{
Development Platforms as a Niche for Software Companies in Open Source Software
}

\author{
Marinette Savonnet ${ }^{1}$, Eric Leclercq ${ }^{2}$, Marie-Noëlle Terrasse ${ }^{3}$, Thierry Grison ${ }^{4}$, \\ George Becker ${ }^{5}$, Anne Sophie Farizy ${ }^{6}$, and Ludovic Denoyelle ${ }^{7}$ \\ 1 LE2I, Université de Bourgogne, France Marinette.Savonnet@u-bourgogne.fr \\ 2 LE2I, Université de Bourgogne, France Eric.Leclercq@u-bourgogne.fr \\ 3 LE2I, Université de Bourgogne, France Marie-Noelle.Terrasse@u-bourgogne.fr \\ ${ }^{4}$ LE2I, Université de Bourgogne, France Thierry. Grison@u-bourgogne.fr \\ ${ }^{5}$ gbecker@nerim.net \\ ${ }^{6}$ ARIST Bourgogne, France \\ 7 ARIST Bourgogne, France 1. denoyelle@bourgogne.cci.fr
}

As long as information systems do not become overly large and while they address a well-known domain, they can be controlled by engineering staff. Nevertheless, when dealing with large-scale, complex, or innovative information systems, it can be difficult to separate design issues and to formulate a meaningful information system proposal. In such a context, platforms for software engineering appear to be a promising approach. In this paper, we propose to view development platforms as a major opportunity for Open Source Software and Open Formats.

One of the major evolutions in the Open Source world is its integration with the proprietary world. Open Source tools and proprietary tools keep mixing up which each other at various levels: on the same machine, on the same company network, on the Internet and more recently even on the same platform (see, e.g., Eclipse [7]). In terms of business strategies, very large companies or very innovative ones enter consortiums for standard definitions. At the same time, small companies offer Open Source products and sell their competency in customizing their products (e.g., technical support, relevant sets of data, fine tuning of the basic software) to a given business context. In both cases, companies now act as service providers rather than as mere producers.

In such a context, development platforms appear to be a sound basis for engineering of flexible products built on consolidation of computer-based solutions and the know-how of users $[2,3,5,6]$. In order to develop such platforms as meaningful industrial products, it is necessary to assure that Open Formats soon become more generally accepted, and completed with exchange and descriptive languages (such as XMI and the MOF for MOF-repositories $[4,8]$ ).

Thus, development platforms need to evolve towards the schema depicted in Figure 1: a description of a business knowledge made available through open formats and plug-ins (either proprietary or Open Source plug-ins). Such platforms can offer (under Open Source licences) basic business-related

Please use the following format when citing this chapter:

Savonnet, M., Leclercq, E., Terrasse, M.-N., Grison, T., Becker, G., Farizy, A.S., and Denoyelle, L., 2006, in IFIP International Federation for Information Processing, Volume 203, Open Source Systems, eds. Damiani, E., Fitzgerald, B., Scacchi, W., Scotto, M., Succi, G., (Boston: Springer), pp. 341-342 


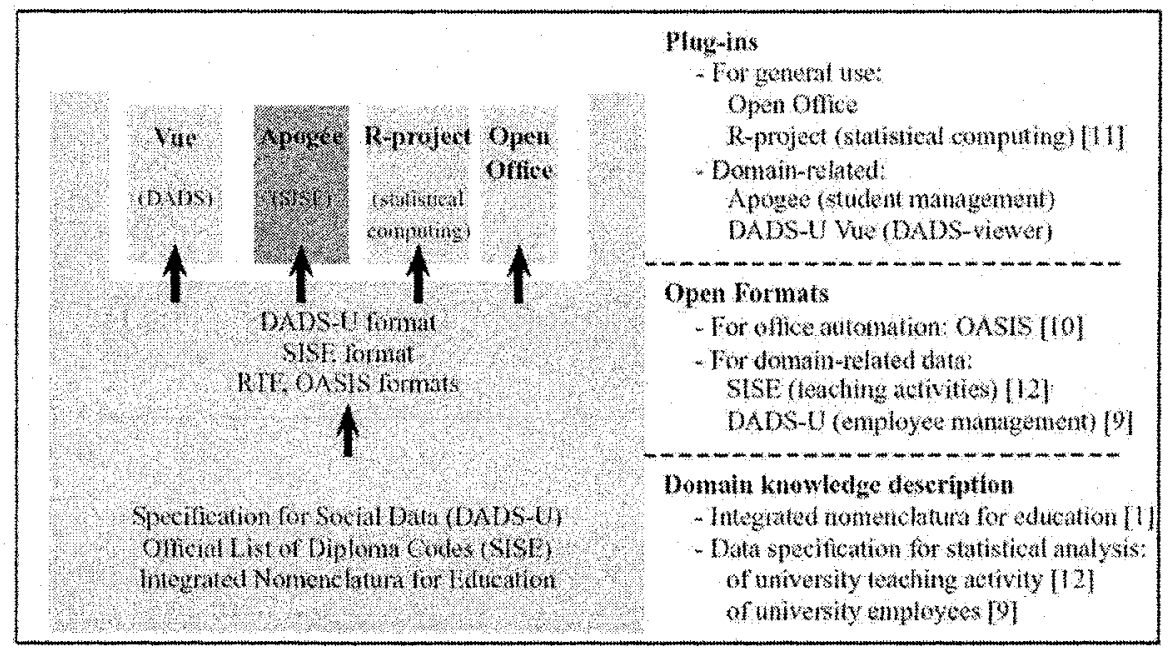

Fig. 1. An example platform for university management

functionalities and can be fine-tuned for specific uses. As an illustration, we describe a platform for education and job market surveys which encompasses: domain knowledge description and specifications, Open Formats, and domain specific plug-ins (Open Source/Format plug-ins are depicted in yellow and proprietary ones in green).

\section{References}

1. Nomenclatures intégrées dans FeDoX (Nomenclatures integrated into $\mathrm{FeDoX}$ ). URL http://fedox.irisa.fr/Pages/nomenclature2.htm.

2. ARIST Bourgogne. Résultats d'enquête sur l'usage de l'informatique et des logiciels libres dans les entreprises bourguignonnes. (Survey Results on the Use of Open Software in Burgundy Companies)

3. J. Koenig. Seven OS Business Strategies for Competitive Advantage. 2005.

4. Meta Object Facility (MOF) Specification, V. 1.4, 2002. URL www.omg.org.

5. T. O'Reilly. OS Paradigm Shift. In Proc. of OSBC'04, 2004.

6. C. Shirky. The Interest Horizons and the Limits of Software Love. 1999.

7. Eclipse Platform. URL http://www.eclipse.org/.

8. XML Metadata Interchange (XMI). URL http://www .omg.org.

9. Déclaration annuelle des données sociales unifiée (DADS-U, Annual Report of Standardized Social Data). URL http://www.travail.gouv.fr/dossiers.

10. OASIS Open Document Format for Office Applications.

11. The R-Project for Statistical Computing. URL http://www.r-project.org/.

12. Programme des opérations statistiques et de contrôle de gestion, SISE. (Program of statistical operations and management inspections) Official Report of the Department of Education, France, 2000. 